\title{
Test of $m_{p} / m_{e}$ changes using vibrational transitions in $\mathrm{N}_{2}{ }^{+}$
}

\author{
Masatoshi Kajita* \\ National Institute of Information and Communications Technology, Koganei, Tokyo 184-8795, Japan
}

Geetha Gopakumar, Minori Abe, and Masahiko Hada

Department of Chemistry, Tokyo Metropolitan University, Hachioji, Tokyo, Japan

Matthias Keller

Department of Physics and Astronomy, University of Sussex, Brighton, United Kingdom

(Received 12 February 2014; published 13 March 2014)

\begin{abstract}
In this paper we propose to utilize the $X^{2} \Sigma_{g}(v, N, F, M)=(0,0,1 / 2, \pm 1 / 2) \rightarrow(1,0,1 / 2, \pm 1 / 2)$ or $(2,0,1 / 2, \pm 1 / 2)$ transition of $\mathrm{N}_{2}{ }^{+}(I=0)$ to test variations of the proton-to-electron mass ratio. The $X^{2} \Sigma_{g}$ ground state exhibits no quadrupole shift and the Zeeman shift of the $N=0 \rightarrow N=0$ transition is exactly zero. Because $\mathrm{N}_{2}{ }^{+}$is nonpolar, systematic level shifts such as Stark shifts induced by trap electric field or blackbody radiation are very small and the thermalization of the rotational states is inhibited. This eases the requirements on the experimental setup significantly. Employing Raman transitions at the "magic" wavelength the $(0,0,1 / 2, \pm 1 / 2) \rightarrow(1,0,1 / 2, \pm 1 / 2)$ or $(2,0,1 / 2, \pm 1 / 2)$ transition frequency can be measured very precisely.

DOI: 10.1103/PhysRevA.89.032509

PACS number(s): 33.20.Ea, 06.30.Ft, 32.60.+i
\end{abstract}

\section{INTRODUCTION}

In 1937, Dirac gave a formulation of the large number hypothesis that aimed to tie together the fundamental constants of physics [1]. Since the measurement uncertainties of atomic transitions became lower than $10^{-16}$ [2-4], testing the variation in the fine-structure constant $\alpha$ has become a "hot topic" in metrology. Rosenband et al. measured the ratio of the ${ }^{27} \mathrm{Al}^{+}{ }^{1} S_{0^{-}}{ }^{3} P_{0}$ and ${ }^{199} \mathrm{Hg}^{+}{ }^{2} S_{1 / 2}{ }^{2} D_{5 / 2}$ transition frequencies and determined that $\alpha$ varies by $(-1.6 \pm 2.3) \times 10^{-17} / \mathrm{yr}[4]$. Another fundamental constant to test is the proton-to-electron mass ratio $m_{p} / m_{e}$, because the ratio of variations in $\alpha$ and $m_{p} / m_{e}$ provides useful information for grand unification theories [5]. Molecular transition frequencies are particularly useful to detect potential variations in $m_{p} / m_{e}$, as vibrational and rotational transition frequencies are to first order proportional to $\left(m_{p} / m_{e}\right)^{-1 / 2}$ and $\left(m_{p} / m_{e}\right)^{-1}$, respectively. van Weerdenburg et al. observed the variation in $m_{p} / m_{e}$ from transition frequencies of $\mathrm{H}_{2}$ molecules in the quasar source J2123-005 (at redshift $z=2.059$ ). Using two different telescopes, variations of $\Delta\left(m_{p} / m_{e}\right) /\left(m_{p} / m_{e}\right)=\left(8.5 \pm 3.6_{\text {stat }} \pm\right.$ $\left.2.2_{\text {syst }}\right) \times 10^{-6}$ and $\left(5.6 \pm 5.5_{\text {stat }} \pm 2.9_{\text {syst }}\right) \times 10^{-6}$ have been found [6]. In contrast, Bagdonaite et al. compared transitions of $\mathrm{CH}_{3} \mathrm{OH}$ molecules from PKS1830-211 (at redshift $z=0.89$ ) with laboratory measurements and obtained a variation of $\Delta\left(m_{p} / m_{e}\right) /\left(m_{p} / m_{e}\right)=(0 \pm 1) \times 10^{-7}[7]$. To distinguish the time and space variations, the astronomical observation must be performed with many objects in different directions across the universe. Using telescopes in the northern and southern hemisphere Webb et al. [8] and King et al. [9] found evidence which indicates spatial as well as temporal variations of $\alpha$.

Laboratory measurements are instrumental in the search for spatial and temporal variations of fundamental constants due to unparalleled control over the environment and the achievable precision of the measurements. In addition, shorter,

*kajita@nict.go.jp well-controlled interrogation times enable the detection of fast changes in fundamental constants. Due to the motion of the Earth, laboratory measurements are sensitive not only to temporal but also to spatial variations. Recently Berengut et al. [10] concluded that the Earth is moving into an area of larger $\alpha$ and thus potentially also larger values of $m_{p} / m_{e}$. From measured ratios between the optical frequencies of atomic transitions and the Cs hyperfine frequency (which is sensitive to variations in both $\alpha$ and $m_{p} / m_{e}$ ) [11-14], the upper limit of the variation in $m_{p} / m_{e}$ is known to be smaller than $10^{-16} / \mathrm{yr}$. To measure the pure variation in $m_{p} / m_{e}$, it is preferable to compare frequencies of molecular transitions with those of atomic transitions, whose dependence on $\alpha$ and $m_{p} / m_{e}$ are negligibly small (e.g., the ${ }^{1} S_{0^{-}}{ }^{3} P_{0}$ transition frequencies of the ${ }^{27} \mathrm{Al}^{+}$ion [4] or ${ }^{87} \mathrm{Sr}$ atom [2,3]). The measured molecular transition must be chosen by considering the sensitivity to $m_{p} / m_{e}$ and achievable accuracy of the measurement. Several authors have presented molecular transition frequencies with sensitivity to $m_{p} / m_{e}$ much higher than pure vibrational or rotational transition frequencies [15-17]. However, these transitions are difficult to measure with an uncertainty lower than $10^{-14}$.

Several neutral molecules have been proposed for testing the variation in $m_{p} / m_{e}$ such as ${ }^{40} \mathrm{Ca}^{6} \mathrm{Li},{ }^{88} \mathrm{Sr}^{6} \mathrm{Li}$ [18], and ${ }^{174} \mathrm{Yb}^{6} \mathrm{Li}[19,20]$. These molecules can be potentially generated in large numbers from cold atoms through photoassociation or Feshbach resonances and may be trapped in optical traps. Collision shifts may be suppressed by trapping the molecules in optical lattices and the ac Stark shift can be eliminated by setting the trap laser frequency to the "magic" wavelength. Although some experimental results have been obtained with ${ }^{40} \mathrm{Ca}^{7} \mathrm{Li}$ molecules in a thermal beam [21,22], generation and trapping of the aforementioned molecules has not been demonstrated thus far.

In contrast to neutral molecules, molecular ions can be easily trapped and cooled through sympathetic laser cooling with atomic ions which are trapped alongside the molecules. With long trapping times in the order of many hours and 
superior isolation from the environment, molecular ions are very strong candidates for precision tests of $m_{p} / m_{e}$ variations. Even though currently the internal molecular states are detected destructively through resonance-enhanced multi-photon ionization (REMPI), resonance-enhanced multi-photon dissociation (REMPD), or light induced charge transfer (LICT), molecular ions offer the possibility to measure the state nondestructively, which promises more accurate spectroscopy. The molecular structures of $\mathrm{H}_{2}^{+}$[23] and $\mathrm{HD}^{+}$[24-26] can be analytically calculated, which makes them good candidates for the measurement of the $m_{p} / m_{e}$ variations. However, the measurement uncertainty is limited by the Stark shift in the $\mathrm{HD}^{+}$transition induced by the trap electric field, because the hyperfine structure is complicated and measurement must be probed with many molecular ions in a Coulomb crystal broadened in all directions. But also other molecular ions have been proposed such as $X^{+}$(with $X$ being the even isotopes of the II group elements) [27] and in particular, ${ }^{40} \mathrm{CaH}^{+}[28,29]$, whose transition frequency measurement can be probed with a single molecular ion in a string crystal, because there is no hyperfine splitting in the rovibrational ground state. The ${ }^{40} \mathrm{CaH}^{+}$vibrational frequency uncertainty is estimated to be in the order of $10^{-16}$. However, due to the permanent electric dipole moment of these molecules, the internal states are constantly reshuffled through the interaction with the blackbody radiation. Even though this has recently been turned into an advantage through blackbody assisted laser cooling of molecules [30], it hampers precision spectroscopy. In order to mitigate the effect of the blackbody radiation, a cryogenic environment is required.

In this paper, we propose to test the variation in $m_{p} / m_{e}$ via the precise measurement of the $(v, N, F, M)=(0,0,1 / 2$, $\pm 1 / 2) \rightarrow(1,0,1 / 2, \pm 1 / 2)(65.2 \mathrm{THz})$ or $(2,0,1 / 2, \pm 1 / 2)$ (129.4 THz) transition [31] of the $X^{2} \Sigma_{g}$ electronic ground state of ${ }^{14} \mathrm{~N}_{2}{ }^{+}(I=0)$ by employing Raman transitions. As a homonuclear diatomic molecule $\mathrm{N}_{2}{ }^{+}$has no $E 1$ transition between different rovibrational states in the $X \Sigma_{g}$ electronic ground state, therefore, the influence of unwanted transitions induced by blackbody radiation is negligibly small. The quadratic dc Stark shift is much smaller than heteronuclear diatomic molecules and precise measurements are possible using molecular ions in a large Coulomb crystal. In addition, the blackbody frequency shift is in the order of $10^{-18}$ and there is no electric quadrupole and Zeeman shift for transitions between rotational ground states. $\mathrm{N}_{2}{ }^{+}$can be sympathetically cooled with laser cooled atomic ions and well localized into the Dicke regime and thus first Doppler shifts can be eliminated. Employing small numbers of molecular ions, a mixed ion string may be cooled into the motional trap ground state and thus even second-order Doppler shifts can be greatly reduced. $\mathrm{N}_{2}{ }^{+}$can be generated by REMPI from neutral nitrogen in a specific rovibrational state where it remains for an extended duration due to its nonpolar structure [32-34]. Therefore, we estimate that precise measurements with uncertainties in the order of $10^{-17}$ can be performed with significantly relaxed constraints on the experimental apparatus compared to heteronuclear diatomic molecules.

We will employ the following notation throughout this paper: $v$ is the vibrational quantum number, $N$ describes the nuclear rotation of the molecule, $I$ is the total nuclear spin of the molecule, $F$ is the hyperfine quantum number and $M_{F}$ is the magnetic quantum number of the hyperfine states, and $S$ denotes the electronic spin of the molecule.

\section{ZEEMAN SHIFT}

In general, energy eigenstates of ${ }^{14} \mathrm{~N}_{2}{ }^{+}$molecular ions with nuclear spin $I$ in the $X^{2} \Sigma_{g}$ state can be described by the wave function $\Psi\left(v, N, F, M_{F}\right)$ which in turn can be expressed by

$$
\Psi=\Sigma a_{m} \psi\left(M_{S}(m), M_{N}(m), M_{I}(m)\right),
$$

with the condition $M_{F}=M_{S}(m)+M_{N}(m)+M_{I}(m)$.

The Zeeman energy shift induced by the magnetic field $B$ is given by

$$
\begin{aligned}
E_{Z}(\Psi)= & h \mu_{B} B\left[g_{S}(\Psi)\left\langle M_{S}(\Psi)\right\rangle\right. \\
& \left.+g_{N}(\Psi)\left\langle M_{N}(\Psi)\right\rangle+g_{I}(\Psi)\left\langle M_{I}(\Psi)\right\rangle\right],
\end{aligned}
$$

with $\left\langle M_{S, N, I}\right\rangle=\Sigma\left|a_{m}\right|^{2} M_{S, N, I}(m)$, the Bohr magneton $\mu_{B}$, and the $g$ factors $g_{S, N, I}$. In general, $a_{m}$ depend on $B$ and the Zeeman shift is not linear. For a low magnetic field, the Zeeman shift can be approximated by

$$
E_{Z}(\Psi) / h=C_{1}(\Psi) B+C_{2}(\Psi) B^{2},
$$

where $C_{1}$ and $C_{2}$ are the first- and second-order Zeeman coefficients. The Zeeman shift of the transition frequency between the states $\Psi_{u}$ and $\Psi_{l}$ is

$$
\begin{aligned}
\Delta f_{Z}= & \frac{E_{Z}\left(\Psi_{u}\right)-E_{Z}\left(\Psi_{l}\right)}{h} \\
\approx & {\left[C_{1}\left(\Psi_{u}\right)-C_{1}\left(\Psi_{l}\right)\right] B } \\
& +\left[C_{2}\left(\Psi_{u}\right)-C_{2}\left(\Psi_{l}\right)\right] B^{2} .
\end{aligned}
$$

In general, there is a significant Zeeman shift in the order of $\mathrm{MHz} / \mathrm{G}$, mainly due to the electron spin. However, for the stretched states $\left(F=S+N+I, M_{F}= \pm F\right),\left\langle M_{S, N, I}\right\rangle$ are all determined to be $\pm S, \pm N, \pm I$ and $E_{Z}$ is strictly proportional to $B$. Because $g_{S}$ has no dependence on the rovibrational state for molecules in the ${ }^{2} \Sigma$ state $(S=1 / 2)$, Eq. (2) can be rewritten as

$$
E_{Z}(\Psi)= \pm \mu_{B} B\left[\frac{1}{2} g_{S}+g_{N}(\Psi) N+g_{I}(\Psi) I\right],
$$

and the Zeeman shift of the $\left(v_{l}, N_{l},\left(I+N_{l}+1 / 2\right), \pm(I+\right.$ $\left.\left.N_{l}+1 / 2\right)\right) \rightarrow\left(v_{u}, N_{u},\left(I+N_{u}+1 / 2\right), \pm\left(I+N_{u}+1 / 2\right)\right)$ transition frequency is given by

$$
\begin{aligned}
\Delta f_{Z}= & \pm \frac{\mu_{B} B}{h}\left\{g_{N}\left(v_{u}\right) N_{u}-g_{N}\left(v_{l}\right) N_{l}\right. \\
& \left.+I\left[g_{I}\left(v_{u}\right)-g_{I}\left(v_{l}\right)\right]\right\} .
\end{aligned}
$$

Actually, $\left|g_{I}\left(v_{u}\right)-g_{I}\left(v_{l}\right)\right| / g_{I}\left(v_{l}\right)$ and $\left|g_{N}\left(v_{u}\right)-g_{N}\left(v_{l}\right)\right| /$ $g_{N}\left(v_{l}\right)$ are in the order of $10^{-7}$ [35] and $10^{-2}$, respectively. Therefore $\Delta f_{Z}$ is

$$
\begin{aligned}
& N_{u} \neq N_{l}: \Delta f_{Z} \approx\left(N_{u}-N_{l}\right) \times 1 \mathrm{kHz} / \mathrm{G}, \\
& N_{u}=N_{l} \neq 0: \Delta f_{Z} \approx N_{l} \times 10 \mathrm{~Hz} / \mathrm{G}, \\
& N_{u}=N_{l}=0: \Delta f_{Z} \approx I \times 0.1 \mathrm{mHz} / \mathrm{G} .
\end{aligned}
$$

This makes $Q(0)$ rovibrational transitions ideal candidates for testing small frequency shifts. ${ }^{14} \mathrm{~N}$ atoms have a nuclear spin of 1 and thus the possible total nuclear spin of the ${ }^{14} \mathrm{~N}_{2}{ }^{+}$ 
molecule is $I=0,1,2$. Due to the symmetry of the nuclear spin wave function, the possible rotational states are severely limited. In the ${ }^{2} \Sigma_{g}$ electronic ground state only even rotational levels are possible for $I=0,2$ and only odd levels for $I=1$. Thus, the $N=0 \rightarrow 0$ transition is observed for molecular ions with $I=0$ and 2 . In consequence, the Zeeman shift of the $\left(v_{l}, 0,1 / 2, \pm 1 / 2\right) \rightarrow\left(v_{u}, 0,1 / 2, \pm 1 / 2\right)$ transitions is exactly zero for $I=0$. While the rotational states can be prepared by REMPI of neutral $\mathrm{N}_{2}$ [32-34], the hyperfine configuration cannot be selected. Therefore, the distinction between $I=0$ (no hyperfine splitting) and 2 (hyperfine splitting of $226 \mathrm{MHz}$ ) is performed from spectroscopic measurements. There is a technical advantage to using the molecular ions with $I=0$, that the choice between $M_{F}= \pm 1 / 2$ is not necessary in the $N=0$ state. It is advantageous to prepare the ${ }^{15} \mathrm{~N}_{2}{ }^{+}$ molecular ion in the $I=0, N=0$ state, because ${ }^{15} \mathrm{~N}$ nuclear spin is $1 / 2$ and the $N=0$ state exists only in the $I=0$ state.

\section{ELECTRIC QUADRUPOLE SHIFT}

In rf ion traps, large electric field gradients can occur which can lead to electric quadrupole shifts of the molecular states. For states with an electric quadrupole moment, this level shift can lead to a significant shift of the transition frequency which in turn hampers high-resolution spectroscopy. An example is the electric quadrupole shift of $S \rightarrow D$ transitions of alkali-metal-like ions [36]. Electric quadrupole shifts exist also for molecular ions in the $N \neq 0$ states. For the $\mathrm{HD}^{+}$ molecular ion, the electric quadrupole coefficients of different rovibrational transitions are estimated to be in the order of $10-150 \mathrm{mHz} /\left(\mathrm{V} / \mathrm{mm}^{2}\right)$ [37], which is one order smaller than that for $S \rightarrow D$ transition of alkali-metal-like ions. The electric quadrupole moment of $\mathrm{N}_{2}{ }^{+}$in its electronic ground state $X^{2} \Sigma_{g}$ is exactly zero for the $I=0$ hyperfine configuration with $N=0$. As a consequence, the $(0,0,1 / 2, \pm 1 / 2) \rightarrow$ $(1,0,1 / 2, \pm 1 / 2)$ or $(2,0,1 / 2, \pm 1 / 2)$ transition frequencies are unaffected by quadrupole shifts.

\section{STARK SHIFT}

In rf ion traps, oscillating fields are employed to confine the ions. Even though the center of the trap is, in principle, field free, ions may be exposed to the trapping electric field. This is especially true, in three-dimensional ion crystals in which ions are not located on the nodal line of a linear ion trap. The electric field experienced by the ions can be significant and may result in a Stark shift of the molecular states. There is no dipole coupling between different rovibrational states in the $X^{2} \Sigma_{g}$ state and thus a second-order Stark shift $\epsilon_{S}(\nu)$ in the $X^{2} \Sigma_{g}(v, N=0)$ state, induced by the oscillating trapping electric field $E=E_{0} \cos (2 \pi f t)$, is given by

$$
\epsilon_{S}(\nu)=S_{v}(f)\left\langle E^{2}\right\rangle_{\mathrm{ave}}=\frac{S_{\nu}(f) E_{0}^{2}}{2},
$$

with

$$
S_{\nu}(f)=-\sum_{\Phi, v^{\prime}} \frac{\mu_{v}\left(\Phi, v^{\prime}\right)^{2}}{h} \frac{f^{\prime}\left(\Phi, v^{\prime}\right)}{f_{v}^{\prime}\left(\Phi, v^{\prime}\right)^{2}-f^{2}},
$$

where $\mu_{v}\left(\Phi, v^{\prime}\right)$ and $f_{v}^{\prime}\left(\Phi, v^{\prime}\right)$ denote the transition dipole moment and transition frequency between the $X^{2} \Sigma_{g}(v, N=$ $0)$ and $\Phi\left(v^{\prime}, N=1\right)$ states $\left(\Phi=A^{2} \Pi_{u}, B^{2} \Sigma_{u}\right.$, and $\left.C^{2} \Sigma_{u}\right)$. The relevant transition parameters are listed in the tables in the Appendix. The Stark shift of the $(v, N)=\left(v_{l}, 0\right) \rightarrow\left(v_{u}, 0\right)$ transition frequency is given by

$$
\Delta f_{S}=\epsilon_{S}\left(v_{u}\right)-\epsilon_{S}\left(v_{l}\right)
$$

Typical ion traps are operated with rf frequencies in the order of $10 s \mathrm{MHz}$. Therefore, the Stark shift can be obtained from Eq. (8) in the limit of $f \rightarrow 0$. The Stark shifts of the upper and lower states canceled to more than $98 \%$ and thus

$$
\frac{\Delta f_{S}}{f_{v}\left(E_{0}^{2} / 2\right)} \approx 8 \times 10^{-20}(\mathrm{~cm} / \mathrm{V})^{2},
$$

where $f_{v}$ denotes the transition frequency from the ground state to the vibrationally excited state $v$. This is much smaller than that for the ${ }^{40} \mathrm{CaH}^{+}$vibrational transition $(-3.1 \times$ $10^{-15}(\mathrm{~cm} / \mathrm{V})^{2}$ [27]). In large three-dimensional ion crystals, the ions are confined in a volume where $E_{0}$ is less than $20 \mathrm{~V} / \mathrm{cm}$ and thus $\Delta f_{S} / f_{v}$ is less than $2 \times 10^{-17}$. Therefore, precise measurement can be performed also with a large Coulomb crystal without being limited by the Stark shift.

\section{BLACKBODY RADIATION SHIFT}

Due to the nonpolar structure, $\mathrm{N}_{2}{ }^{+}$has no $E 1$ allowed rotational transitions in its electronic ground state. This not only inhibits blackbody induced redistribution of rotational states but also greatly reduces the effect of blackbody radiation on the transition frequencies. The blackbody radiation shift $\Delta f_{B}$ of the vibrational transition from the ground state to the vibrationally excited state $v$ induced by blackbody radiation at ambient temperature $T$ is given by

$$
\frac{\Delta f_{B}}{f_{\nu}} \approx \frac{1}{f_{\nu}} \int \frac{8 \pi h f^{3}}{\varepsilon_{0} c^{3}} \frac{S_{\nu}(f)-S_{0}(f)}{\exp \left(h f / k_{B} T\right)-1} d f .
$$

The resulting frequency shift $\Delta f_{B} / f_{v}$ at $T=300 \mathrm{~K}$ is estimated to be $4 \times 10^{-18}$. This low shift is the result of the low transition dipole moment of transitions to the lowest electronically excited state $A^{2} \Pi$. Furthermore, the blackbody shift is partly compensated due to the close spacing of the $v=0,1$, and 2 levels. The low blackbody radiation shift makes it viable to measure the transition frequency with high precision even without a cryogenic system.

\section{RAMAN LASER INDUCED STARK SHIFT}

In order to measure the $v=0 \rightarrow 1$ or 2 transition precisely, rotational ground state $Q(0)$ transitions should be employed. However, direct $Q(0)$ transitions are forbidden even in higher transition order. Thus the $X^{2} \Sigma_{g}(\nu, N)=(0,0) \rightarrow(\nu, 0)$ transition frequency should be measured by probing the two-photon Raman transition with two lasers with intensities $I_{R 0}$ and $I_{R v}$ and frequencies $f_{R 0}$ and $f_{R v}=f_{R 0}-f_{\nu}$. The Rabi frequency of this Raman transition $\Omega_{R} / 2 \pi$ is 
TABLE I. Magic Raman frequencies $f_{R 0}$ and $f_{R \nu}=f_{R 0}-f_{c}$ for which the Stark shift in the $v=0 \rightarrow 1$ and 2 transition frequency $\left(\delta f_{R} / f_{c}\right)$ is zero at equal power densities of the two Raman lasers. Also listed are power densities of both lasers $I_{R}$, where the transition Rabi frequency $\Omega_{R} / 2 \pi$ corresponds to $1 \mathrm{~Hz}$.

\begin{tabular}{lccc}
\hline \hline & $f_{R 0}(\mathrm{THz})$ & $f_{R v}(\mathrm{THz})$ & $I_{R}\left(\mathrm{~W} / \mathrm{cm}^{2}\right)$ \\
\hline$v=0 \rightarrow 1$ & 541.6 & 476.4 & 3 \\
$v=0 \rightarrow 2$ & 421.1 & 291.7 & 2 \\
\hline \hline
\end{tabular}

given by

$$
\frac{\Omega_{R}}{2 \pi}=\frac{\sqrt{I_{R 0} I_{R v_{u}}}}{\varepsilon_{0} c h^{2}} \sum_{\Phi, v^{\prime}} \frac{\mu_{0}\left(\Phi, v^{\prime}\right) \mu_{v_{u}}\left(\Phi, v^{\prime}\right)}{f_{0}^{\prime}\left(\Phi, v^{\prime}\right)-f_{R 0}} .
$$

These Raman lasers cause an ac Stark shift of the states and thus can cause systematic shifts of the transition frequency. The Stark shift induced by the Raman lasers is given by

$$
\Delta f_{R}=\Delta f_{R 0}+\Delta f_{R \nu},
$$

with

$$
\Delta f_{R n}=\frac{I_{R n}}{\varepsilon_{0} c}\left[S_{v}\left(f_{R n}\right)-S_{0}\left(f_{R n}\right)\right] \quad(n=0, v),
$$

where $S_{0, v}\left(f_{R n}\right)$ is given by Eq. (8). By choosing the laser wavelength correctly, the contributions for the various excited states to the level shift cancel and thus the effect of the Raman laser on the transition frequency can be eliminated $[19,20,29,33]$. At this magic wavelength, only differential changes in the laser intensities may cause a shift of the transition frequency. The magic wavelength of the $v=0 \rightarrow 1$ and 2 transitions are listed in Table I for $I_{R 0}=I_{R v}$. For a Rabi frequency of the Raman transition of $\Omega_{R}=1 \mathrm{~Hz}$, fluctuations of $1 \%$ in the laser intensities cause a relative Stark shift on the order of $10^{-17}$. This effect can be further reduced by using the hyper Ramsey method [38].

\section{CONCLUSION}

In this paper we proposed to test the time variation of the electron-to-proton mass ratio through high-resolution spectroscopy of the $Q(0) v=0 \rightarrow 1$ or 2 transition of the ${ }^{14} \mathrm{~N}_{2}{ }^{+}(I=0)$ electronic ground state. We have estimated all relevant shifts of the transition frequency such as the blackbody radiation, dc Stark, and laser induced shifts and have determined that the Zeeman and quadrupole shift of the
$M_{F}= \pm F \rightarrow \pm F$ transitions vanish. All the investigated systematic shifts of the relevant transitions in $\mathrm{N}_{2}{ }^{+}$are smaller than those of other proposed molecular ions. A direct comparison with $\mathrm{CaH}^{+}$and $\mathrm{HD}^{+}$is shown in Table II. Due to its nonpolar structure, $\mathrm{N}_{2}{ }^{+}$has a significantly smaller blackbody and dc Stark shift. This, together with the high sensitivity of the transition to changes in $m_{e} / m_{p}$ (defined by $\left.\left[\Delta f_{v} / f_{v}\right] /\left[\Delta\left(m_{p} / m_{e}\right) /\left(m_{p} / m_{e}\right)\right]\right)$ of 0.49 (ideal case: 0.5 ) and the vanishing Zeeman and quadrupole shifts, makes $\mathrm{N}_{2}{ }^{+}$ a prime candidate for measuring changes in the electronto-proton mass ratio. Note also that the natural linewidth is much narrower than $0.1 \mathrm{~Hz}$, because there is no $E 1$ transition between the rovibrational states in the $X^{2} \Sigma_{g}$ state. Apart from these fundamental spectroscopic properties, the experimental requirements are also significantly simpler for $\mathrm{N}_{2}{ }^{+}$than for $\mathrm{CaH}^{+}$and $\mathrm{HD}^{+}$. Due to the absence of a permanent electric dipole moment, the rotational states are protected against thermalization through interaction with the blackbody radiation. Thus having prepared the molecular ions in a specific state, it remains there for long times [39], in principle only limited by the collision with neutral $\mathrm{N}_{2}$ from the background gas. $\mathrm{N}_{2}{ }^{+}$ions can be easily generated through REMPI of neutral $\mathrm{N}_{2}$ and thus ions can be loaded into the trap within seconds. Employing a pulsed molecular beam, the background gas level can be negligible. Due to its charge-to-mass ratio, $\mathrm{N}_{2}{ }^{+}$can be efficiently cooled with laser cooled calcium ions. Since all lasers for the high-resolution spectroscopy of $\mathrm{N}_{2}{ }^{+}$ and to cool the calcium ions are available as diode lasers, the laser system can be comparably cheap and compact. Furthermore, the ${ }^{40} \mathrm{Ca}^{+}$ions can be employed to detect the internal state of $\mathrm{N}_{2}{ }^{+}$using quantum logic spectroscopy [4,34] or state selective dipole forces [25]. With a low sensitivity of the $S_{1 / 2} \rightarrow D_{5 / 2}$ quadrupole transition to variations in the fine-structure constant and $m_{e} / m_{p}$, the calcium ions can serve as a reference for the $\mathrm{N}_{2}{ }^{+}$transition frequency. In addition, the calcium ions trapped alongside the molecular ions can serve as a probe to monitor environmental parameters. In summary, we have demonstrated that $\mathrm{N}_{2}{ }^{+}$is a very suitable candidate to search for variations of the electron-to-proton mass ratio.

\section{APPENDIX}

The transition dipole moment $\mu_{v}\left(\Phi, v^{\prime}\right)$ and transition frequency $f_{v}^{\prime}\left(\Phi, v^{\prime}\right)$ between the $X^{2} \Sigma_{g}(v, N=0)$ and $\Phi\left(v^{\prime}, N=\right.$ 1) states $\left(\Phi=A^{2} \Pi_{u}, B^{2} \Sigma_{u}\right.$, and $\left.C^{2} \Sigma_{u}\right)$ are shown in Tables III and IV.

TABLE II. Comparison of $(v, N)=(0,0)-(2,0)$ transition of ${ }^{14} \mathrm{~N}_{2}{ }^{+},{ }^{40} \mathrm{CaH}^{+}$, and $\mathrm{HD}^{+}$molecular ions. Listed are the relative frequency shifts of the transition.

\begin{tabular}{lccc}
\hline \hline & ${ }^{14} \mathrm{~N}_{2}{ }^{+}$ & ${ }^{40} \mathrm{CaH}^{+}[29]$ & $\mathrm{HD}^{+}[37]$ \\
\hline Transition freq. $(\mathrm{THz})$ & 129.4 & 85.6 & 112 \\
Natural linewidth (Hz) & $<0.1$ & 5.5 & 0.14 \\
Relative Zeeman shift $\left(\mathrm{G}^{-1}\right)$ & 0 & $1.3 \times 10^{-18}$ & 0 \\
dc Stark shift $(\mathrm{cm} / \mathrm{V})^{2}$ & $8 \times 10^{-20}$ & $-3 \times 10^{-15}$ & 0 \\
Quadrupole shift $\left(\mathrm{mm}^{2} / \mathrm{V}\right)$ & 0 & $-6 \times 10^{-16}$ & 0 \\
Blackbody shift $(300 \mathrm{~K})$ & $4 \times 10^{-18}$ & 0.48 & $1.1 \times 10^{-17}$ \\
Sensitivity $\left(\left[\Delta f_{v} / f_{v}\right] /\left[\Delta\left(m_{p} / m_{e}\right) /\left(m_{p} / m_{e}\right)\right]\right)$ & 0.49 & 0.47 \\
\hline \hline
\end{tabular}


TABLE III. The $X^{2} \Sigma_{g}(v, N)=(v, 0) \rightarrow \Phi(v, N)=\left(v^{\prime}, 1\right)$ transition dipole moments $\mu_{v}\left(\Phi, v^{\prime}\right)[31]$ are listed $\left(\Phi: A^{2} \Pi_{u}, B^{2} \Sigma_{u}\right.$, and $\left.C^{2} \Sigma_{u}\right)$.

\begin{tabular}{llllllll}
\hline \hline & $v^{\prime}=0$ & $v^{\prime}=1$ & $v^{\prime}=2$ & $v^{\prime}=3$ & $v^{\prime}=4$ & $v^{\prime}=5$ & $v^{\prime}=6$ \\
\hline$\mu_{0}\left(A^{2} \Pi_{u}, v^{\prime}\right)(\mathrm{D})$ & 0.26 & 0.22 & 0.15 & 0.085 & 0.046 & 0.025 & 0.013 \\
$\mu_{1}\left(A^{2} \Pi_{u}, v^{\prime}\right)(\mathrm{D})$ & 0.22 & 0.069 & 0.18 & 0.18 & 0.13 & 0.026 & 0.050 \\
$\mu_{2}\left(A^{2} \Pi_{u}, v^{\prime}\right)(\mathrm{D})$ & 0.12 & 0.21 & 0.053 & 0.11 & 0.16 & 0.15 & 0.007 \\
$\mu_{0}\left(B^{2} \Sigma_{u}, v^{\prime}\right)(\mathrm{D})$ & 0.86 & 0.56 & 0.19 & 0.035 & 0.00002 & 0.00011 & 0.00014 \\
$\mu_{1}\left(B^{2} \Sigma_{u}, v^{\prime}\right)(\mathrm{D})$ & 0.56 & 0.51 & 0.62 & 0.28 & 0.058 & 0.0030 & 0.0039 \\
$\mu_{2}\left(B^{2} \Sigma_{u}, v^{\prime}\right)(\mathrm{D})$ & 0.30 & 0.59 & 0.24 & 0.62 & 0.35 & 0.074 & 0.010 \\
$\mu_{0}\left(C^{2} \Sigma_{u}, v^{\prime}\right)(\mathrm{D})$ & 0.021 & 0.037 & 0.047 & 0.050 & 0.047 & 0.042 & 0.00009 \\
$\mu_{1}\left(C^{2} \Sigma_{u}, v^{\prime}\right)(\mathrm{D})$ & 0.058 & 0.076 & 0.067 & 0.044 & 0.020 & 0.0013 & 0.015 \\
$\mu_{2}\left(C^{2} \Sigma_{u}, v^{\prime}\right)(\mathrm{D})$ & 0.11 & 0.086 & 0.032 & 0.015 & 0.038 & 0.040 & 0.0069 \\
\hline \hline
\end{tabular}

TABLE IV. $X^{2} \Sigma_{g}(v, N)=(v, 0) \rightarrow \Phi(v, N)=\left(v^{\prime}, 1\right)$ transition frequencies $f_{v}^{\prime}\left(\Phi, v^{\prime}\right)[31]$ are listed $\left(\Phi: A^{2} \Pi_{u}, B^{2} \Sigma_{u}\right.$, and $\left.C^{2} \Sigma_{u}\right)$.

\begin{tabular}{|c|c|c|c|c|c|c|c|c|}
\hline & $v^{\prime}=0$ & $v^{\prime}=1$ & $v^{\prime}=2$ & $v^{\prime}=3$ & $v^{\prime}=4$ & $v^{\prime}=5$ & $v^{\prime}=6$ & $v^{\prime}=7$ \\
\hline$f_{0}^{\prime}\left(A^{2} \Pi_{u}, v^{\prime}\right)(\mathrm{THz})$ & 270.3 & 326.4 & 381.7 & 436.1 & 489.5 & 542.1 & 593.8 & 644.5 \\
\hline$f_{1}^{\prime}\left(A^{2} \Pi_{u}, v^{\prime}\right)(\mathrm{THz})$ & 205.1 & 261.2 & 316.5 & 370.9 & 424.3 & 476.9 & 528.6 & 579.3 \\
\hline$f_{2}^{\prime}\left(A^{2} \Pi_{u}, v^{\prime}\right)(\mathrm{THz})$ & 140.9 & 197.0 & 252.3 & 306.7 & 360.1 & 412.7 & 464.4 & 515.1 \\
\hline$f_{0}^{\prime}\left(B^{2} \Sigma_{u}, v^{\prime}\right)(\mathrm{THz})$ & 766.4 & 837.5 & 907.0 & 974.7 & 1040.5 & 1104.1 & 1165.2 & 1223.5 \\
\hline$f_{1}^{\prime}\left(B^{2} \Sigma_{u}, v^{\prime}\right)(\mathrm{THz})$ & 701.2 & 772.3 & 841.8 & 909.5 & 975.3 & 1038.9 & 1100.0 & 1158.3 \\
\hline$f_{2}^{\prime}\left(B^{2} \Sigma_{u}, v^{\prime}\right)(\mathrm{THz})$ & 637.0 & 708.1 & 777.6 & 845.3 & 911.1 & 974.7 & 1035.8 & 1094.1 \\
\hline$f_{0}^{\prime}\left(C^{2} \Sigma_{u}, v^{\prime}\right)(\mathrm{THz})$ & 1934.8 & 1996.4 & 2057.2 & 2117.3 & 2176.5 & 2234.9 & 2292.2 & 2348.6 \\
\hline$f_{1}^{\prime}\left(C^{2} \Sigma_{u}, v^{\prime}\right)(\mathrm{THz})$ & 1869.6 & 1931.2 & 1992.0 & 2052.1 & 2111.3 & 2169.7 & 2227.0 & 2283.4 \\
\hline$f_{2}^{\prime}\left(C^{2} \Sigma_{u}, v^{\prime}\right)(\mathrm{THz})$ & 1805.4 & 1866.9 & 1927.8 & 1987.8 & 2047.1 & 2105.5 & 2162.8 & 2219.1 \\
\hline
\end{tabular}

[1] P. A. M. Dirac, Nature (London) 139, 323 (1937).

[2] M. M. Boyd, A. D. Ludlow, S. Blatt, S. M. Foreman, T. Ido, T. Zelevinsky, and J. Ye, Phys. Rev. Lett. 98, 083002 (2007).

[3] M. D. Swallows, M. Bishof, Y. Lin, S. Blatt, M. J. Martin, A. M. Rey, and J. Ye, Science 331, 1043 (2011).

[4] T. Rosenband, D. B. Hume, P. O. Schmidt, C. W. Chou, A. Brusch, L. Lorini, W. H. Oskay, R. E. Drullinger, T. M. Fortier, J. E. Stalnaker, S. A. Diddams, W. C. Swann, N. R. Newbury, W. M. Itano, D. J. Wineland, and J. C. Bergquist, Science 319, 1808 (2008).

[5] X. Calmet and H. Fritzsch, Eur. Phys. J. C 24, 639 (2002).

[6] F. van Weerdenburg, M. T. Murphy, A. L. Malec, L. Kaper, and W. Ubachs, Phys. Rev. Lett. 106, 180802 (2011).

[7] J. Bagdonaite, P. Jansen, C. Henkel, H. L. Bethlem, K. M. Menten, and W. Ubachs, Science 339, 46 (2013).

[8] J. K. Webb, J. A. King, M. T. Murphy, V. V. Flambaum, R. F. Carswell, and M. B. Bainbridge, Phys. Rev. Lett. 107, 191101 (2011).

[9] J. A. King, J. K. Webb, M. T. Murphy, V. V. Flambaum, R. F. Carswell, M. B. Bainbridge, M. R. Wilczynska, and F. E. Koch, Mon. Not. R. Astron. Soc. 422, 3370 (2012).

[10] J. C. Beregut and V. V. Flambaum, Europhys. Lett. 97, 20006 (2012).

[11] V. V. Flambaum, Int. J. Mod. Phys. A 22, 4937 (2007).

[12] V. V. Flambaum, D. B. Leinweber, A. W. Thomas, and R. D. Young, Phys. Rev. D 69, 115006 (2004).
[13] V. V. Flambaum and A. F. Tedesco, Phys. Rev. C 73, 055501 (2006).

[14] T. H. Dinh, A. Dunning, V. A. Dzuba, and V. V. Flambaum, Phys. Rev. A 79, 054102 (2009).

[15] H.L. Bethlem, M. Kajita, B. Sartakov, G. Meijer, and W. Ubachs, Eur. Phys. J.: Spec. Top. 163, 55 (2008).

[16] V. V. Flambaum and M. G. Kozlov, Phys. Rev. Lett. 99, 150801 (2007).

[17] D. DeMille, S. Sainis, J. Sage, T. Bergeman, S. Kotochigova, and E. Tiesinga, Phys. Rev. Lett. 100, 043202 (2008).

[18] M. Kajita, G. Gopakumar, M. Abe, and M. Hada, J. Phys. B 46, 025001 (2013).

[19] M. Kajita, G. Gopakumar, M. Abe, and M. Hada, Phys. Rev. A 84, 022507 (2011).

[20] M. Kajita, G. Gopakumar, M. Abe, and M. Hada, Phys. Rev. A 85, 062519 (2012).

[21] A. Ivanova, A. Stein, A. Pashov, A. V. Stolyarov, H. Knoeckel, and E. Tiemann, J. Chem. Phys. 135, 174303 (2011).

[22] G. Krois, J. V. Pototshinig, F. Lackner, and W. Ernst, J. Phys. Chem. A 117, 13719 (2013).

[23] L. Hilico, N. Billy, B. Gremaud, and D. Delande, J. Phys. B 34, 491 (2001).

[24] J. C. J. Koelemeij, B. Roth, A. Wicht, I. Ernsting, and S. Schiller, Phys. Rev. Lett. 98, 173002 (2007).

[25] J. C. J. Koelemeij, B. Roth, and S. Schiller, Phys. Rev. A 76, 023413 (2007). 
[26] T. Schneider, B. Roth, H. Duncker, I. Ernsting, and S. Schiller, Nat. Phys. 6, 275 (2010).

[27] M. Kajita, M. Abe, M. Hada, and Y. Moriwaki, J. Phys. B 44, 025402 (2011).

[28] M. Kajita and Y. Moriwaki, J. Phys. B 42, 154022 (2009).

[29] M. Kajita and M. Abe, J. Phys. B 45, 185401 (2012).

[30] P. F. Staanum, K. Hojbjerre, P. S. Skyt, A. K. Hansen, and M. Drewsen, Nat. Phys. 6, 271 (2010).

[31] F. R. Gilmore, R. R. Laher, and P. J. Espy, J. Phys. Chem. Ref. Data 21, 1005 (1992).

[32] S. R. Mackenzie, F. Merkt, E. J. Halse, and T. P. Softley, Mol. Phys. 86, 1283 (1995).
[33] T. Zanon-Willette, A. D. Ludlow, S. Blatt, M. M. Boyd, E. Arimondo, and J. Ye, Phys. Rev. Lett. 97, 233001 (2006).

[34] X. Tong, D. Wild, and S. Willitsch, Phys. Rev. A 83, 023415 (2011).

[35] G. Gopakumar, M. Abe, M. Hada, and M. Kajita, Phys. Rev. A 84, 045401 (2011).

[36] W. M. Itano, J. Res. Natl. Inst. Stand. Technol. 105, 829 (2000).

[37] D. Bakalov and S. Schiller, arXiv:1307.4340v2.

[38] V. I. Yudin, A. V. Taichenachev, C. W. Oates, Z. W. Barber, N. D. Lemke, A. D. Ludlow, U. Sterr, Ch. Lisdat, and F. Riehle, Phys. Rev. A 82, 011804(R) (2010).

[39] J. Mur-Petit, J. J. Garcia-Ripoll, J. Perez-Rios, J. CamposMartinez, M. I. Hernandez, and S. Willitsch, Phys. Rev. A 85, 022308 (2012). 